\title{
BMJ Open Estimation of perinatal mortality rate for institutional births in Rajasthan state, India, using capture-recapture technique
}

\author{
Prem K Mony, ${ }^{1}$ Beena Varghese, ${ }^{1,2}$ Tinku Thomas ${ }^{1}$
}

To cite: Mony PK, Varghese B, Thomas T. Estimation of perinatal mortality rate for institutional births in Rajasthan state, India, using capturerecapture technique. $B M J$ Open 2015;5:e005966. doi:10.1136/bmjopen-2014005966

- Prepublication history for this paper is available online. To view these files please visit the journal online (http://dx.doi.org/10.1136/ bmjopen-2014-005966).

Received 15 July 2014 Revised 15 January 2015 Accepted 16 January 2015

CrossMark

\footnotetext{
${ }^{1}$ Division of Epidemiology, Biostatistics \& Population Health, St John's Research Institute, Bangalore, Karnataka, India

${ }^{2}$ Public Health Foundation of India, New Delhi, India
}

Correspondence to Dr Prem K Mony; prem_mony@sjri.res.in

\section{ABSTRACT}

Objective: The objective of our investigation was to estimate the perinatal mortality rate among institutional births and to compare the sensitivities of different data collection methods.

Setting: A hospital-based prospective cohort study was undertaken during late-2012 in 21 public sector health facilities of 10 districts of the northern state of Rajasthan, India.

Participants: A total of 6872 births were included in this epidemiological study.

Primary and secondary outcome measures:

Perinatal mortality rate of institutional births was the primary outcome. Sensitivities of 'active' and passive' data collection methods were the secondary outcome measures.

Methods: All stillbirth data were from routine government records ('passive system'); early neonatal outcome data from government records ('passive') were compared against the method of 'phone-tracking' of outcomes through the community health worker ('active system'). The Lincoln-Petersen formula for capture-recapture method was used to calculate the probable missing number of early neonatal deaths and thereby estimate the institutional perinatal mortality rate.

Results: Ratio of births in district:subdistrict facilities was $55: 45$. The estimated perinatal mortality rate $(95 \%$ $\mathrm{CI}$ ) by capture-recapture method was 35.8 (34 to 37) per 1000 births. The sensitivity of the passive system was $87-89 \%$ while the sensitivity of the active system was $91 \%$. Three-fourths of perinatal deaths were documented as stillbirths. However, for these reported intrauterine deaths or stillbirths, clinical classification by typologies (term vs preterm; intrapartum vs antepartum; macerated vs fresh; with or without congenital anomalies) was absent in the recording system.

Conclusions: Capture-recapture technique can be used to estimate the institutional perinatal mortality rate and also to assess the level of under-reporting by the 'passive' government reporting system. This can subsequently be used for monitoring of trends and studying the impact of health interventions. Accurate clinical categorisation of perinatal deaths is also recommended for improving quality of care.
Strengths and limitations of this study

- Routine government reporting systems do not facilitate calculation of perinatal mortality rate (PNMR) for institutional births.

- Capture-recapture (CR) technique enables estimation of PNMR for institutional births and also the level of under-reporting by 'passive' government reporting systems.

- CR-derived estimate of PNMR can be used as baseline to monitor trends and impact of interventions.

- While early neonatal deaths were captured by two different methods, late fetal deaths (stillbirths) were obtained only from a single source in our study.

\section{INTRODUCTION}

The federal government of India, in 2005, launched the National Rural Health Mission (NRHM), a flagship programme committed to increasing public spending on health from $0.9 \%$ to $2-3 \%$ of Gross Domestic Product (GDP) annually. ${ }^{1}$ Innovations under this programme including conditional cash transfers for institutional deliveries (Janani Suraksha Yojana (JSY)), emergency transport, recruitment of over 800000 village health volunteers (Accredited Social Health Activists (ASHA)) nationwide, 24/7 functioning of rural primary health centres, hiring of contractual staff and provision of essential obstetric/neonatal care, have led to a substantial increase $(\sim 30 \%)$ in institutional deliveries over a few years across the country. ${ }^{1-4}$ The next level of investment is to follow-up on building staff capacity and improving the quality of care in healthcare facilities across the country. ${ }^{12}$ This step requires critical information on health system inputs, processes and outcomes for meaningful interpretation of the impact of maternal, fetal and neonatal health services. Perinatal mortality rate is one such key health outcome; therefore its accurate definition and documentation (late fetal deaths and 
early neonatal deaths) would be an important first step. The ascertainment of perinatal deaths is, however, difficult. Routine health information systems suffer from incorrect reporting of perinatal events owing to incomplete capture, absent or improper linkage, poor case definitions, inadequate demarcation of catchment population, emphasis on passive reporting without supporting validation studies and inadequate clinical documentation. ${ }^{56}$ Multiple data sources may be available for documenting maternal, delivery and perinatal outcomes of institutional births. Information on the reporting completeness of such different systems is essential for policymakers and programme managers to make optimal use of available data capture systems.

Capture-recapture (CR) methods may be useful for estimating the true frequency of reported plus unreported cases of a disorder in a population. This method is based on matching two or more independent samples to arrive at an estimate of the total number of diseases/ events when both of the lists are potentially incomplete but with a definite overlap. Although initially used in the field of wildlife biology, over the past few decades it has been adopted in demographic and epidemiological studies. ${ }^{78}$ It is now widely used in developed as well as developing country settings for several disorders that are incompletely captured through epidemiological surveillance. ${ }^{9-12}$ The validity of the estimates relies on four key assumptions: (1) the population being studied must be 'closed', (2) individuals must be matched reliably, (3) sources should be independent and (4) the chance of being 'captured' by a source must be equal. ${ }^{13} 14$

In the northwestern state of Rajasthan, in India, the local state government with technical support from Jhpiego-India, is implementing an adapted version of the WHO's Safe Childbirth Checklist (SCC) programme as a tool to improve adherence to evidence-based practices at four different 'pause-points' around childbirth-on arrival of woman in the delivery room; before pushing/ operative delivery; after delivery and before discharge from hospital. ${ }^{15}$ This 29-item, checklist-based quality improvement programme is being evaluated through a quasi-experimental trial design in 200 public sector health facilities including district hospitals (DHs), subdistrict hospitals (SDHs) and community health centres (CHCs) across 13 districts of the state during 2012-2015. In this communication, we describe the estimation of the perinatal mortality rate for institutional births in select hospitals of Rajasthan state and compare the sensitivities of different data capture methods.

\section{METHODS}

Study design

Prospective cohort study.

\section{Study setting}

Basic sociodemographic and health indicators for the state of Rajasthan in the year 2011 were: total population $=69$ million; total number of districts $=33$; total fertility rate $=3.1$; maternal mortality ratio $=331$ per 1000 live births; infant mortality ratio $=60$ per 1000 live births; female literacy rate $=53 \%$; and proportion of institutional births in government hospitals was 56\%. ${ }^{16-18}$ Out of 200 study sites, 21 facilities with high-volume deliveries (contributing to $25 \%$ of births) and distributed across 10 districts were purposively selected for this study (figure 1).

\section{Data collection systems}

Information on deliveries was collected from all these 21 high-delivery-load facilities (nine DHs, one SDH and 11 CHCs) for the 4-week period, 1-30 November 2012, and from 12 of these facilities (5 DHs, $1 \mathrm{SDH}$ and 6 CHCs) for a further 2-week period, 1-15 December 2012. This yielded a total of 11020 births (8085 births in November and 2935 births in December; figure 1). Information on deliveries was collected from two sources-labour room (LR) and operation theatre (OT) registers-in all facilities. It included mother's identifier data (name, age, address and husband's name), obstetric details (parity, gestational age (GA), type of delivery and number of newborns), newborn details (sex, birthweight, date and time of delivery) and birth outcomes (live birth or stillbirth). The proportion of births in DHs was 62\% (6849/ $11020)$ and that in SDH/CHCs was 38\% (4171/11 020).

Information on perinatal outcomes was collected using two independent methods-passive and active methods. In both methods, information on stillbirths was obtained 'passively' from the labour room/operation theatre registers. The difference was in data collection on early neonatal deaths (ENDs). In the 'passive' method, information was collected 'passively' from three different routine sources-labour room/postnatal ward (LR) registers and newborn care (NC) unit $^{\mathrm{i}}$ registers for institutional ENDs and from Monthly Death Registers (MDRs) for out-of-institutional ENDs. The first two registers were maintained within the health facilities while the MDRs were collated at the district level from reports by community health workers (auxiliary nurse midwives (ANMs)). Among institutional deaths, only 'inborn' deaths, that is, those deaths among babies born in the corresponding facility $(\mathrm{DH} / \mathrm{CHC})$ were included in our linkage analysis while deaths among sick newborns who were 'referred in' from other hospitals or home births were not.

In the 'active' method, information was obtained 'actively by phone-tracking' at the end of seven days. This involved a team of research assistants who made telephone calls during 1 December 2012 to 15 February 2013 to all community-based ANMs in these 10 districts for individual tracking of the status of live births at the end of the early neonatal period. The ANMs responsible for a population of 5000 each provided updates directly or after checking with the ASHA volunteers working

${ }^{\mathrm{i}}$ Called Facility-Based Newborn Care (FBNC) units or NewBorn Stabilisation Units (NBSU) in Rajasthan. 
Figure 1 Flow diagram

depicting the districts, health facilities and births covered in the study.

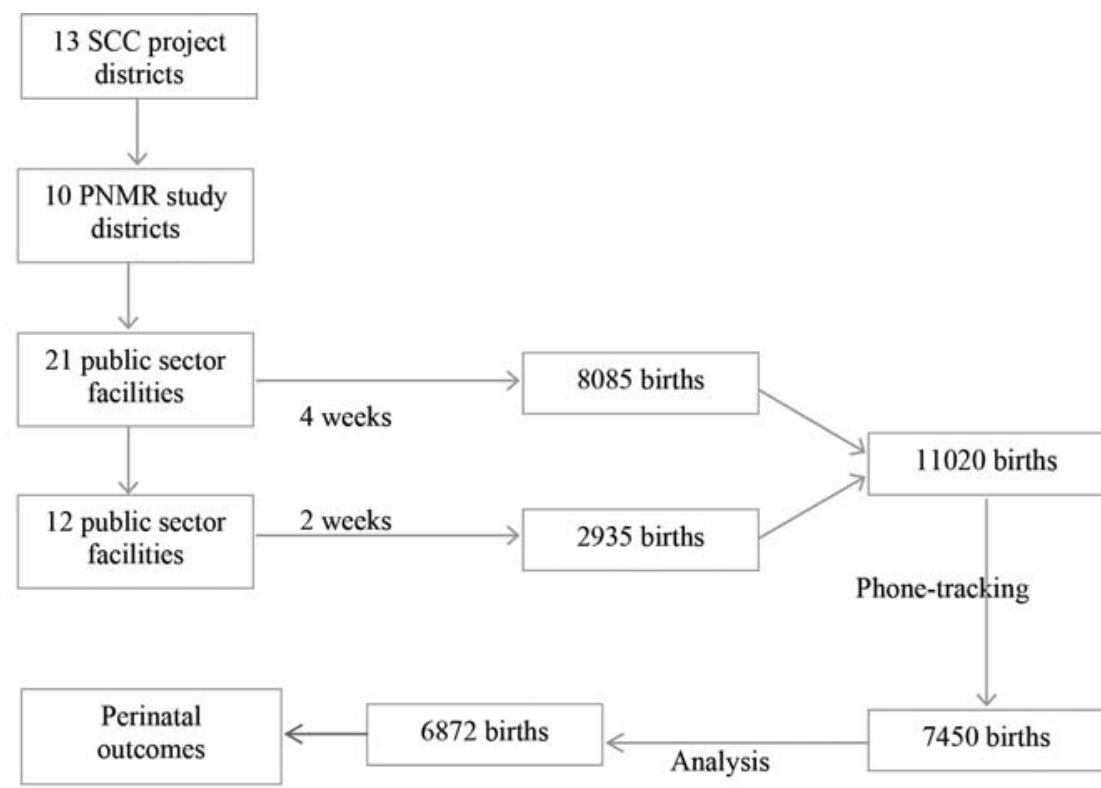

Key: $\mathrm{SCC}=$ Safe Childbirth Checklist; PNMR = Perinatal mortality rate; births = stillbirths \& livebirths

under them and covering a population of approximately 1000 each. A total of 7450 (68\%) of 11020 births were tracked successfully over telephone; $61 \%$ of births in DHs and $78 \%$ of births in SDH/CHCs. Out of the total of 7450 births that were covered by the 'active' plus 'passive' methods, 6872 births had adequate information (on birthweight, gestational age and newborn status) and so were included in the analysis. This sample size had $80 \%$ power to detect a PNMR of 30 per 1000 at $20 \%$ precision and $\mathrm{p}=0.01$ level of significance.

In addition, for all cases of ENDs reported by the ANMs, we set up a validation substudy by making an additional phone call directly to the village ASHA volunteer to corroborate the vital status of the baby.

\section{Analysis}

Given the absence of a unique identification number for linking mothers and newborns listed in the different registers, matching was carried out individually by a combination of mother's identifier details (name, age, husband's name, address) and childbirth information (type of delivery, date of birth, sex of baby). The Lincoln-Petersen unbiased formula was used to estimate the true number of perinatal deaths $(\mathrm{N})=\left[\left(\mathrm{N}_{\mathrm{A}}+1\right) \times\left(\mathrm{N}_{\mathrm{B}}+1\right) /\left(\mathrm{N}_{\mathrm{AB}}+1\right)\right]-1$, wherein $\mathrm{N}_{\mathrm{A}}$ denotes the number of deaths captured by the 'passive' government registers system, $\mathrm{N}_{\mathrm{B}}$ denotes the number of deaths captured by the 'active' phone-tracking system and $\mathrm{N}_{\mathrm{AB}}$ denotes the number of deaths captured by both systems (table 1). ${ }^{7} 13$ The $95 \%$ CI for this CR estimate was also calculated. ${ }^{9}$

Multiple register sources were collapsed into a simpler 2-register method $(\mathrm{LR}+\mathrm{NC})$ or 3-register method (LR+NC+MDR) for comparison against the phonetracking method. PNMR was calculated for the '2-register' method (excluding community-based MDR), '3-register' method, phone-tracking method and the CR method.

PNMR was calculated using standard definition; the denominator was total births (live births and stillbirths) and the numerator was perinatal deaths that included both reported stillbirths and reported early neonatal deaths (deaths up to 7 days). ${ }^{5}$ Stillbirth was defined as late fetal death at or after 28 weeks' gestation (or) weighing $>1000 \mathrm{~g}$ at birth. ${ }^{19}$ Sensitivity was calculated using standard technique as the proportion of the total estimated perinatal deaths captured by each method.

To model dependence between sources, separate loglinear models were constructed with main effects of different sources alone and different combinations of two-way interactions. ${ }^{20}$

\section{RESULTS}

\section{Estimation of PNMR}

Ratio of DH:non-DH births was 55:45. The number of stillbirths recorded by the passive system of the government labour room registers was 182 .

The total yield of early neonatal deaths from 2 registers was $33\left(\mathrm{~N}_{\mathrm{A}}=\mathrm{N}_{\mathrm{AB}}+\mathrm{a}=21+12\right)$ and from phone tracking

\begin{tabular}{|c|c|c|c|}
\hline & \multicolumn{3}{|c|}{ Source B (phone tracking) } \\
\hline & Yes & No & \\
\hline \multicolumn{4}{|c|}{ Source A (registers) } \\
\hline Yes & $\mathrm{N}_{\mathrm{AB}}$ & $\mathrm{a}$ & $\mathrm{N}_{\mathrm{A}}$ \\
\hline No & $b$ & $?$ & \\
\hline Total & $\mathrm{N}_{\mathrm{B}}$ & $\mathrm{N}$ & \\
\hline
\end{tabular}


Table 2 Calculation of institutional perinatal mortality rate by different surveillance methods

\begin{tabular}{llllll}
\hline $\begin{array}{l}\text { Surveillance } \\
\text { Method }\end{array}$ & $\begin{array}{l}\text { Stillbirths } \\
\text { (N) }\end{array}$ & $\begin{array}{l}\text { Neonatal } \\
\text { deaths (N) }\end{array}$ & $\begin{array}{l}\text { Perinatal } \\
\text { deaths (N) }\end{array}$ & $\begin{array}{l}\text { Births } \\
\text { tracked (N) }\end{array}$ & $\begin{array}{l}\text { PNMR/1000 } \\
\text { (95\% Cl) }\end{array}$ \\
\hline Register method & & & & & \\
$\quad$ 2-registers & 182 & 33 & 215 & 6872 & $31.3(30.1$ to 32.6) \\
$\quad$ 3-registers & 182 & 37 & 219 & 6872 & $31.9(30.4$ to 33.3) \\
$\begin{array}{l}\text { Phone-tracking method } \\
\text { Cap-recap method }\end{array}$ & 182 & 41 & 223 & $6872(30.8$ to 34.0) & 32.6 \\
\hline
\end{tabular}

PNMR, perinatal mortality rate; 2-registers, labour room and newborn care unit registers; 3-registers, labour room, newborn care unit and monthly death registers (latter from community).

was $41\left(\mathrm{~N}_{\mathrm{B}}=\mathrm{N}_{\mathrm{AB}}+\mathrm{b}=21+20\right)$ neonatal deaths. Using both methods yielded $53\left(\mathrm{~N}=\mathrm{N}_{\mathrm{AB}}+\mathrm{a}+\mathrm{b}=21+12+20\right)$ deaths. The total number of estimated early neonatal deaths worked out to a total of 64 by the CR method (with 11 being missed by both). Hence the total number of perinatal deaths was 246 (182 stillbirths and 64 early neonatal deaths).

Table 2 illustrates the capture of perinatal deaths by the different methods (registers vs phone tracking) and the estimated numbers by the CR method in order to compute the PNMR of institutional births. The 95\% CI for the estimate by the CR method is also shown.

The stillbirth rate was 26.5 per 1000; and the Early Neonatal Mortality rate calculated by the three different methods were: 5.5 per 1000 (registers method), 6.1 per 1000 (phone-tracking method) and 9.6 per 1000 (cap-recap method).

\section{Sensitivity of different surveillance methods}

Sensitivity of each of the different methods in capturing early neonatal deaths or perinatal deaths with the cap-recap method as the reference group is shown in table 3 .

\section{Other maternal and perinatal characteristics}

Mean age of mothers was 25 years. Proportion of births by caesarean section was $5 \%$. Singleton deliveries was $98 \%$ and twin deliveries were $2 \%$. Gestational ages (GA) were universally recorded only in weeks and not in weeks+days. In most facilities, there was no variability in values with all GA values being 36,38 or 40 weeks only; in a handful of facilities where there were other values recorded, it was noted that it was mostly between 35 and 40 and not the full range between 28 and 42 weeks.
There was marked digit preference noted in the recording of birthweights of newborns, especially for values of $2.00,2.25,2.50,2.75,3.00$ and $3.5 \mathrm{~kg}$. Low birthweight $(<2.5 \mathrm{~kg})$ prevalence was $18 \%$.

In addition, it was also noted that for reported intrauterine deaths or stillbirths, adherence to consistent and uniform clinical classification of stillbirths by typologies (term vs preterm; intrapartum vs antepartum; macerated vs fresh; with or without congenital anomalies) was absent in the recording system.

Lastly, in log-linear regression analysis, the model with the interaction of phone tracking and newborn care (NC) register gave the best fitting model. The three-way interaction was not valid for these data.

\section{DISCUSSION}

Perinatal deaths remain invisible in global policies though they are mostly preventable. Lack of adequate data on perinatal deaths hampers visibility, effective policies and research. Simply counting would be the first step in analysis and prevention. Use of the CR method provides an opportunity to estimate the unreported events based on the fact that there could be two or more independent systems with a partial overlap. The use of this method in our study has provided a reasonably robust estimate of PNMR of 35.8 per 1000 for institutional births in these select hospitals in Rajasthan, which would otherwise have not been possible from the prevailing incomplete reporting systems. Our estimate was much higher than the 17 per 1000 recorded in a series of 10892 deliveries in a hospital in the north Indian state of Chhattisgarh ${ }^{21}$ and slightly lower than the rate of 40.6 per 1000 noted in a series of 31007 births from across 21 centres of the National Neonatal

Table 3 Sensitivities of different methods in capturing early neonatal and all perinatal deaths

\begin{tabular}{|c|c|c|c|c|}
\hline \multirow[b]{2}{*}{ Method } & \multicolumn{2}{|c|}{ Early neonatal deaths } & \multicolumn{2}{|c|}{ Perinatal deaths } \\
\hline & $\bar{n} / \mathrm{N}$ & Per cent & $\bar{n} / \mathrm{N}$ & Per cent \\
\hline \multicolumn{5}{|l|}{ Registers method } \\
\hline 2-registers & $33 / 64$ & 52 & $215 / 243$ & 87 \\
\hline 3-registers & $37 / 64$ & 58 & $219 / 243$ & 89 \\
\hline Phone-tracking method & $41 / 64$ & 64 & $223 / 243$ & 91 \\
\hline
\end{tabular}

2-registers, labour room and newborn care unit registers; 3-registers, labour room, newborn care unit and monthly death registers; $n$, number captured by this method; N, number estimated by capture-recapture method. 
Perinatal Database, ${ }^{22}$ which included stillbirths as those over the age of 20 weeks' gestation.

Of the two surveillance methods that we used, the 'phone tracking through community health workers' method yielded a slightly higher number of early neonatal deaths $(n=41)$ than that obtained by the routine government records (33 ENDs by the 2-register method and 37 by the 3-register method). Compared to the institutional registers, phone tracking yielded nearly a $25 \%$ higher estimate of ENDs. However, when stillbirths and neonatal deaths were considered together, the difference between the 3-register method and phone-tracking method was $\sim 2 \%$.

Clubbing data from the two registers (labour room and NC unit registers) available in the healthcare facility yielded lower numbers than those obtained when additionally considering the monthly death register, which also captures home-based deaths from among institutional births. The additional yield in Rajasthan was about $7 \%$ for ENDs and 1\% for all perinatal deaths; this was probably because of differential roll-out of the community-based data capture system across districts and also possible time-delays between the occurrence and reporting of these community-based deaths. Decisions on including such community-based death reporting systems will therefore be context-dependent after quantification of the relative contribution of different sources.

Depending on the definition used, fetal mortality usually contributes to about $40-60 \%$ of perinatal mortality. ${ }^{53}$ However, in our study, it was seen that about $75 \%$ of all perinatal deaths among the institutional deliveries in Rajasthan were recorded as stillbirths with only $25 \%$ of outcomes being recorded as early neonatal deaths. This may be due to substantial misclassification of early neonatal deaths as stillbirths or may be due to suboptimal healthcare practices surrounding the time of delivery/childbirth. ${ }^{5} 624{ }^{25}$ We believe that it may be a function of both; it was found that in several healthcare facilities, deaths of newborns occurring within a few minutes (up to possibly an hour) after birth were routinely reported as stillbirths rather than as ENDs.

In addition, it was also noted that certain critical clinical characteristics that need to be captured regarding institutional deliveries that could potentially be used for facility-level interventions aimed at optimising perinatal outcomes were inadequately captured. There was no standard and uniform reporting methodology being followed for classification of stillbirths/perinatal deaths regarding: (1) term or preterm, (2) antepartum or intrapartum-related, (3) macerated or fresh stillbirths and (4) whether there was presence or absence of gross congenital anomalies. There were also inadequacies in the reporting of gestational age of mothers and birthweights of newborns. Gestational age was routinely reported only in weeks (and not in weeks+days) with median values clustering around 36 with no variability; and birthweight recording showed excessive rounding- off to a few select values in multiples of $0.25 \mathrm{~kg}$ between 2.00 and $3.50 \mathrm{~kg}$. Recording of these critical data accurately would yield salient information that is potentially actionable at the facility level for improving the quality of care through interventions such as the Safe Childbirth Checklist and others. ${ }^{15} 2325$

We also examined if the assumptions of CR method were satisfied. ${ }^{13}{ }^{14}$ First, our study involved a closed population-it was limited to all births within a 6-week period in a defined set of healthcare facilities. Second, there was homogeneity of capture-that is, for a given source, each death had the same 'probability' of being captured. Third, there was correct linkage based on a combination of 'matching variables' (consisting of maternal and newborn characteristics) that were individually matched in a meticulous manner. Last, we believe that the different sources were independent by design; the 'passive' government registers (labour room, $\mathrm{NC}$ unit and the community registers) and our 'active' phone tracking were gathered by separate reporting systems and were not mutually exclusive or complementary. However, log-linear regression analysis indicated that this was not completely so, with a possibility of positive dependence between the $\mathrm{NC}$ unit register and phone-tracking method. This will, however, at the worst, lead to under-estimation of the perinatal mortality rate ${ }^{26}$ the true rate of PNMR would therefore be greater than our study estimate. In addition, there is a possible limitation in our study of some misclassification between 'late fetal deaths' ( $>28$ weeks GA) and early fetal deaths (particularly those occurring between 24 and 28 weeks GA) owing to deficiencies in correct estimation of gestational ages in these facilities.

Two types of biases-selection and reporting biasare relevant in studies such as ours. ${ }^{27}$ The choice of hospitals (at district or subdistrict level) with different 'case-mix' of mothers/newborns is likely to affect maternal and neonatal outcomes. Further, within hospitals it is known that phone ownership is linked to the socioeconomic status of clients. However, in our study, this was not an issue because we did not seek phone numbers of families, but instead telephoned the health workers regarding neonatal outcomes. However, with regard to coverage, it is important to note that we were able to obtain information on $68 \%$ (7450/11 020) only, with differential success being noted dependent on residence (rural CHCs $=78 \%$ vs urban DHs=61\%) due to incomplete documentation of residential addresses in the latter. Families that are harder-to-reach are usually more likely those with higher mortality experience and so our estimate of PNMR is, at best, likely an under-estimate. There are possibly three different biases in the identification of medical conditions by families: recognition bias, recall bias and reporting bias. However, in our study, the outcome status (alive or dead) was not likely to have been misclassified due to recognition or recall bias. The validation substudy helped us to infer that there 
was no misclassification in the reporting of neonatal outcomes by the phone-tracking method.

A key limitation of our CR study was that all stillbirth data were from a unique source (government registers) and only the neonatal outcome data were obtained from two different sources. However, because of the probable misclassification of a substantial number of early neonatal deaths as stillbirths in this setting, restricting our study to only early neonatal deaths would have yielded a falsely low rate of early neonatal mortality. Hence we considered it prudent to club early neonatal deaths with the stillbirths to yield a more meaningful PNMR estimate.

In summary, the institutional perinatal mortality rate in these select government hospitals of Rajasthan was estimated to be 35.8 per 1000 births by 'capturerecapture' technique. Phone tracking through health workers yielded $91 \%$ of estimated perinatal deaths compared to $87-89 \%$ from routine government records. Once such an estimate of the institutional PNMR is calculated and the proportion of under-reporting by the 'passive' government reporting system is known, the latter could still be useful for purposes of routine monitoring of trends and studying the impact of institutional interventions on perinatal deaths. In addition, more accurate classification of stillbirths versus early neonatal deaths, better capture of intrapartum-related perinatal deaths by documenting fetal heart rate at arrival, better classification of macerated or fresh stillbirths and documentation of presence or absence of congenital anomalies will go a long way in paving the way for improving the quality of care. Implementation of simple, proven interventions in maternal and perinatal care, and relevant operational research, ${ }^{23}{ }^{25}$ along with improved monitoring, will help enhance and document improved perinatal health in resource-limited settings in the near future.

Acknowledgements The authors are grateful to Rajashree Panicker, Shibumi Prem, Neha Dumka, Akash Kumar and Puneet Srivastav (study team at PHFI office in Jaipur) for assistance in data collection and to Kiruba Sankar for reviewing an earlier version of the manuscript.

Contributors PKM, BV and TT conceived and designed the study. PKM and BV conducted the study. PKM, BV and TT performed the analysis. PKM wrote the manuscript, and BV and TT reviewed the manuscript. PKM is the guarantor for the study.

Funding This work was supported by The Children's Investment Fund Foundation (CIFF), UK and UBS Optimus Foundation, Switzerland.

Competing interests None.

Ethics approval Permission for the overall trial was approved by the government of Rajasthan and facilitated through a Technical Advisory Group (TAG). Ethical approval for the study was obtained from the Institutional Ethics Committee of the Public Health Foundation of India, New Delhi, India (TRC-IEC-141/12; 28 Sept 2012).

Provenance and peer review Not commissioned; externally peer reviewed.

Data sharing statement No additional data are available.

Open Access This is an Open Access article distributed in accordance with the Creative Commons Attribution Non Commercial (CC BY-NC 4.0) license, which permits others to distribute, remix, adapt, build upon this work non-commercially, and license their derivative works on different terms, provided the original work is properly cited and the use is non-commercial. See: http://creativecommons.org/licenses/by-nc/4.0/

\section{REFERENCES}

1. National Rural Health Mission. Common review mission. New Delhi, India: Ministry of Health and Family Welfare, Govt of India, 2011. http://www.mohfw.nic.in/NRHM/CRM/CRM files/ltem 3CRM.htm (accessed 2 Dec 2011).

2. Lim SS, Dandona L, Hoisington JA, et al. India's Janani Suraksha Yojana, a conditional cash transfer programme to increase births in health facilities: an impact evaluation. Lancet 2010;375:2009-23.

3. Husain Z. Health of the National Rural Health Mission. Econ Polit Wkly 2011;22;46:53-60.

4. Singh SK, Kaur R, Gupta M, et al. Impact of national rural health mission on perinatal mortality in rural India. Indian Pediatr 2012;49:136-8.

5. Barfield WD; Committee on fetus and newborn. Standard terminology for fetal, infant, and perinatal deaths. Pediatrics 2011;128:177-81

6. Merrill RM. Reproductive epidemiology: principles and methods. USA: Jones and Bartlett Publishers, 2010:77-107.

7. LaPorte RE, Barinas E, Chang YF, et al. Global epidemiology and public health in the 21st century: applications of new technology. Ann Epidemiol 1996;6:162-7.

8. Laska EM. The use of capture-recapture methods in public health Bull World Health Organ 2002;80:845.

9. Kiakalayeh AD, Mohammadi R, Stark Ekman D, et al. Estimating drowning deaths in northern Iran using capture-recapture method. Health Policy 2011;100:290-6.

10. deSá J, Alcalde-Cabero E, Almazán-Isla J, et al. Capture-recapture as a potentially useful procedure for assessing prevalence of multiple sclerosis: methodologic exercise using Portuguese data. Neuroepidemiology 2012;38:209-16.

11. Khazaei S, Poorolajal J, Mahjub H, et al. Estimation of the frequency of intravenous drug users in Hamadan city, Iran, using the capture-recapture method. Epidemiol Health 2012;34:e2012006.

12. Huang WT, Huang WI, Huang YW, et al. The reporting completeness of a passive safety surveillance system for pandemic (H1N1) 2009 vaccines: a capture-recapture analysis. Vaccine 2012;30:2168-72.

13. International Working Group for Disease Monitoring and Forecasting Capture-recapture and multiple-record systems estimation. II. Applications in human diseases. Am J Epidemiol 1995;142:1059-68.

14. University of Pittsburgh. Capture-recapture web-page. USA 2013 http://www.pitt.edu/ yuc2/cr/main.htm (accessed 30 Jan 2013).

15. World Health Organization. Safe childbirth checklist programme: an overview. WHO/HIS/PSP/2013.1. Switzerland: World Health Organization, 2013.

16. Registrar General of India. Census Rajasthan. New Delhi, India: Ministry of Home Affairs, Govt of India, 2011. http://censusindia.gov. in/2011-prov-results/prov_data_products_rajasthan.html (accessed 30 Jul 2013).

17. Registrar General of India. Sample registration system -SRS bulletin vol. 46, no.1. New Delhi, India: Ministry of Home Affairs, Govt of India, 2011. http://censusindia.gov.in/vital_statistics/SRS Bulletins/SRS_Bulletin_December_2011.pdf (accessed 22 Aug 2013).

18. Registrar General of India. Annual health survey. Indicators for 2010-11. New Delhi, India: Ministry of Home Affairs, Govt of India, 2011. http://data.gov.in/dataset/annual-health-survey (accessed 29 Dec 2012).

19. World Health Organization. Maternal, neonatal, child and adolescent health. Stillbirths. Switzerland: World Health Organization, 2013. http://www.who.int/maternal_child_adolescent/epidemiology/stillbirth/ en/ (accessed 12 Aug 2013).

20. Bohning D. Capture-recapture procedures in public health and social sciences, 2010. http://www.personal.soton.ac.uk/dab1f10/ Capture-Recapture-muenster.pdf (accessed 20 Sep 2013).

21. Singh A, Nandi L. Obstetric emergencies: role of obstetric drill for a better maternal outcome. J Obstet Gynaecol India 2012;62:291-6.

22. NNPD Network. National neonatal-perinatal database report 200203. New Delhi, India: Department of Pediatrics, All India Institute of Medical Sciences, 2005. 
23. Moss W, Darmstadt GL, Marsh DR, et al. Research priorities for the reduction of perinatal and neonatal morbidity and mortality in developing country communities. J Perinatol 2002;22:484-95.

24. World Health Organization. Neonatal and perinatal mortality: country, regional and global estimates. Switzerland: World Health Organization, 2006. http://whqlibdoc.who.int/ publications/2006/9241563206_eng.pdf (accessed 19 Dec, Aug 2013).
25. Stanton C, Lawn JE. The epidemiology of stillbirths and neonatal deaths. In: Hussein J, McCaw-Binns A, Webber R, eds. Maternal and perinatal health in developing countries. Oxfordshire, UK: CABI Publishing, 2012:51-63.

26. Bierrenbach A. Capture-recapture. WHO/STB/TME, 2009. http://www. who.int/tb/advisory_bodies/impact_measurement_taskforce/meetings/ ie_jul09_capture_recapture.pdf (accessed 20 Sep 2013).

27. Delgado-Rodriguez M, Llorca J. Bias. J Epidemiol Community Health 2004;58:635-41. 